\title{
A Framework for Planning Comfortable and Customizable Motion of an Assistive Mobile Robot
}

\author{
Shilpa Gulati ${ }^{1}$
}

\author{
Chetan Jhurani ${ }^{2}$
}

\author{
Benjamin Kuipers ${ }^{3}$
}

\begin{abstract}
Assistive mobile robots that can navigate autonomously can greatly benefit people with mobility impairments. Since an assistive mobile robot transports a human user from one place to another, its motion should be comfortable for human users. Moreover, it should be possible for users to customize the motion according to their comfort. While there exists a large body of work on motion planning for mobile robots, very little attention has been paid to characterizing comfort and planning comfortable trajectories.

In this paper, we first characterize comfortable motion by formulating a measure of discomfort as a weighted sum of the total travel time and time integrals of various kinematic quantities. We then present a method for factoring the weights such that once a user has customized the weights for one task, the same choice of weights leads to similar average value of the discomfort measure in other tasks.

We seek trajectories that minimize the discomfort and satisfy boundary conditions on pose, velocity and acceleration. Such a problem can naturally be formulated as a variational optimization problem. Unlike previous work, we present a comprehensive formulation that allows the travel time to be unspecified and includes boundary conditions on position, orientation, velocity and acceleration. This makes the formulation very general as it can be used to compute trajectories for various kinds of tasks, such as starting from rest, coming to rest, moving from one specified velocity to another, arriving at a goal with a specified orientation etc. Finally, we present a fast and robust numerical method for solving the minimization problem.
\end{abstract}

\section{INTRODUCTION}

An assistive mobile robot that transports a human user has to perform many tasks that require it to travel from an initial state to a goal state with specified pose, velocity and acceleration. For example, to dock at a user's desk, the robot must arrive at the desk with zero velocity and acceleration and position and align itself correctly. For any motion task, the trajectory of the robot should not only satisfy boundary conditions, it should also result in motion that is comfortable for human users. Moreover, the motion should be customizable to suit an individual user's comfort level. In this paper, we formally characterize comfortable motion and develop a framework for planning customizable and

1,4 gulati@mail.utexas.edu,r.longoria@mail.utexas.edu Department of Mechanical Engineering

The University of Texas at Austin

2 chetan@ices.utexas.edu

Institute for Computational Engineering and Sciences

The University of Texas at Austin

3 kuipers@umich.edu

Computer Science and Engineering

University of Michigan comfortable trajectories. By a trajectory, we mean the robot pose as a function of time.

Measures of discomfort based on the peak, average and root mean squared values of acceleration and jerk, are used in the design of road [1] and railway vehicles [2], [3]. For nonholonomic mobile robot trajectories, continuity of velocity and acceleration is considered important and much research has focused on planning smooth paths and subsequent time-scaling to find such trajectories [4], [5], [6], [7], [8], [9], [10]. In the neuroscience literature, it has been shown that smooth point-to-point reaching movements of human arm can be replicated by minimizing the time integral of the squared $L^{2}$ norm of the hand jerk over the motion duration [11], [12]. Minimum jerk models were also used for planning smooth trajectories of manipulators [13] and time-scaling of paths for nonholonomic mobile robots [7].

Motivated by this, and by the requirement that the motion be as fast as is consistent with comfort, we formulate a measure of discomfort as a weighted sum of the following terms: travel time and time integrals of the squares of tangential jerk, normal jerk, angular velocity, and angular acceleration. Jerk is separated into tangential and normal components to allow different weights for the two components. This is because normal jerk may contribute more to motion discomfort than tangential jerk [3]. In addition, large angular velocity and acceleration also contribute to discomfort. The objective is to include all the terms that contribute to user discomfort and give the user the flexibility to decide their relative importance by changing the weights.

Instead of choosing the weights manually, we use dimensional analysis [14] to partially determine the weights. Each weight is factored into two parts. The first part has physical units and is a fixed function of the length and velocity scales. The second part is a dimensionless multiplicative factor that can be customized by the user. Since the customizable part of a weight is dimensionless, it will result in similar average value of discomfort measure in other tasks with different length and velocity scales.

Given a set of weights, we seek trajectories that satisfy the boundary conditions on pose, velocity and acceleration, and minimize the discomfort. Such a problem can naturally be cast in a variational calculus framework [15]. Variational methods have been widely applied to trajectory planning in robotics, aerospace engineering and control-systems engineering, primarily in the form of optimal control. The problem formulation consists of constructing a cost func- 
tional representing the cumulative cost over the duration of motion, and minimizing the cost functional to find a desired trajectory.

Variational formulations for trajectory planning of wheeled mobile robots have focused primarily on minimum-time trajectories. These trajectories result in geometric paths that consist of a sequence of straight-line and arc segments [16], [17], [18]. These paths do not have curvature continuity and the robot cannot be driven on these paths with smooth velocity controls. Thus, minimum-time trajectories are of little practical use for assistive robots where comfort is important. When other performance measures are used, several limiting assumptions are made, such as known travel time, or boundary conditions on position but not orientation, or boundary conditions on pose but not its derivatives etc. [19], [13], [20], [21], [22]. In this paper, we do not make these limiting assumptions, and present a comprehensive formulation of trajectory planning for a nonholonomic mobile robot as a variational minimization problem that allows the travel time to be unspecified and includes boundary conditions on position, orientation, velocity and acceleration.

Numerically, a variational optimization problem is solved by either indirect or direct methods [23]. Indirect methods solve the first order differential equations representing the necessary conditions of optimality. One frequently used method is the "shooting method" [21], [24]. This method is very sensitive to the initial guess of the unknown parameters, and a poor initial guess sometimes results in "wild" trajectories with values exceeding the numerical range of the computer [23]. Direct methods discretize the problem to convert it into a finite-dimensional problem that is solved using nonlinear optimization techniques. These are generally more robust than indirect methods. Direct methods have been used for planning trajectories for manipulator arms [13], [20] and nonholonomic robots [19], [22]. In our framework, we use a direct method for a numerical solution. We choose a heptic spline discretization and use Newton trust-region method to solve the resulting finite-dimensional problem.

Simulation results show fast convergence toward a minimum. The characteristic weights determined using dimensional analysis result in similar values of the discomfort measure for different boundary conditions. Results also show that the root mean squared value of the various discomfort terms decrease uniformly (as a power law) as the multiplicative factors for the appropriate weights are increased.

We summarize our key contributions here: First, we recognize that motion of an assistive mobile robot should be comfortable and formulate a measure of discomfort in terms of its state variables. Since the notion of comfort is subjective, the measure of discomfort is formulated as a weighted sum of relevant quantities where the weights serve as customizable parameters. Second, we use dimensional analysis to determine weights as functions of characteristic length and velocity scales. Weights computed in this way result in similar values of the discomfort measure for different boundary conditions. Users can choose an appropriate multiplicative factor according to their comfort. Third, we present a comprehensive and general formulation of trajectory planning as a variational minimization problem and describe a fast numerical method for computing trajectories in near real-time.

This paper lays down the groundwork for developing an algorithm in the future that will use human evaluations of trajectories to customize the weights. This first attempt toward generating customizable trajectories will undergo future refinements as comfort level experiments with human users are conducted in the laboratory.

\section{TRAJECTORY PLANING AS A VARIATIONAL MINIMIZATION PROBLEM}

Given a starting pose and an end pose, our objective is to find a motion that satisfies the boundary conditions and minimizes the cost functional. This is done by reducing the discomfort of the user while keeping a small travel time. The discomfort is modeled by a cost functional $J$, which is a function of the total travel time and motion as parameterized by time. This section defines the cost functional, provides rationale for various choices, and discusses the method for numerical solution.

\section{A. The cost functional}

For a robot moving on a planar curve, $\mathbf{r}(t)=(x(t), y(t))$ denotes the position vector at time $t$ (Figure 1). The unit tangent and normal vectors, $\mathbf{T}$ and $\mathbf{N}$, are given by

$$
\mathbf{T}=\frac{\dot{\mathbf{r}}}{\|\dot{\mathbf{r}}\|}, \quad \mathbf{N}=\frac{\dot{\mathbf{T}}}{\|\dot{\mathbf{T}}\|}
$$

The dot represents derivative with respect to $t$. The angle $\theta$ that the tangent makes with the $x$ axis is given by

$$
\theta=\operatorname{atan} 2(\dot{y}, \dot{x})
$$

The mathematical expressions for $\mathbf{T}, \mathbf{N}$, and $\theta$ above are meaningful if and only if the tangential speed is non-zero. We will discuss in Section II-C how these can be defined when tangential speed is zero.

We model the robot as a rigid body moving in a plane. We assume that the $x$-axis of its body-centered coordinate frame is always tangent to the curve $\mathbf{r}(t)$. Hence, $\theta$, as given in Equation 2, is sufficient to represent the orientation of the robot, and the nonholonomic constraint $\dot{x} \sin \theta-\dot{y} \cos \theta=0$ is automatically satisfied.

Let the robot start from $\mathbf{r}_{0}$ at $t=0$ with known speed $v_{0}$, angle $\theta_{0}$, and tangential acceleration $a_{0}$, and reach $\mathbf{r}_{\tau}$ at $t=\tau$ with known speed $v_{\tau}$, angle $\theta_{\tau}$, and tangential acceleration $a_{\tau}$. The travel time $\tau$ is not known yet.

We construct a cost functional $J$ that reflects the trade-off between the travel time and smoothness of motion. The goal is to find a function $\mathbf{r}(t)$ and a travel time $\tau$ that minimize $J$. Since $\dot{\theta}$ and $\ddot{\theta}$ can be determined from $\dot{\mathbf{r}}$ and its higher derivatives (Equation 2), $J$ depends only on $\mathbf{r}$ and $\tau$.

$$
\begin{aligned}
J=\tau & +w_{T} \int_{0}^{\tau}(\dddot{\mathbf{r}} \cdot \mathbf{T})^{2} d t+w_{N} \int_{0}^{\tau}(\dddot{\mathbf{r}} \cdot \mathbf{N})^{2} d t \\
& +w_{\dot{\theta}} \int_{0}^{\tau} \dot{\theta}^{2} d t+w_{\ddot{\theta}} \int_{0}^{\tau} \ddot{\theta}^{2} d t
\end{aligned}
$$




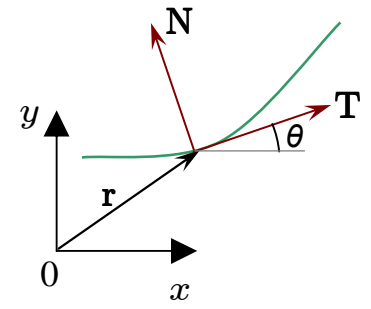

Fig. 1. Tangent and Normal to a curve

Here $\dddot{\mathbf{r}}$ represents the jerk. $\dddot{\mathbf{r}} \cdot \mathbf{T}$ and $\dddot{\mathbf{r}} \cdot \mathbf{N}$ are the tangential and normal components of jerk respectively. $\dot{\theta}$ is the angular velocity and $\ddot{\theta}$ is the angular acceleration. We assume that $\mathbf{r}(t)$ is smooth enough for the cost functional to be welldefined. This means (at least) that the acceleration vector is continuous and normal and tangential components of jerk are square integrable.

The term $\tau$ is necessary. If it is not included in the functional, the optimal solution is to reach the destination at $\tau=\infty$ traveling at essentially zero speed in the limit (except perhaps at the end-points where the speed is already specified). Thus, minimizing just the integral terms will not lead to a good solution.

The weights $\left(w_{T}, w_{N}, w_{\dot{\theta}}, w_{\ddot{\theta}}\right)$ are non-negative known real numbers. We separate tangential and normal jerk to allow a choice of different weights $\left(w_{T}\right.$ and $\left.w_{N}\right)$. The terms with $\dot{\theta}$ and $\ddot{\theta}$ provide independent control over the angular velocity and acceleration.

The weights serve two purposes. Second, they determine the relative importance of the terms and provide a way to adjust the robot's performance according to user preferences. For example, for a wheelchair, some users may not tolerate high jerk and prefer traveling slowly while others could tolerate relatively high jerks if they reach their destination quickly. We will choose characteristic weights using dimensional analysis in Section II-E.

\section{B. The optimization problem}

Find a function $\mathbf{r}$ and a scalar $\tau$ that minimize $J$ given the boundary conditions

$$
\begin{array}{llll}
\mathbf{r}(0) & =\mathbf{r}_{0}, & \mathbf{r}(\tau) & =\mathbf{r}_{\tau} \\
\theta(0) & =\theta_{0}, & \theta(\tau) & =\theta_{\tau} \\
\dot{\mathbf{r}}(0) & =v_{0} \mathbf{q}_{0}, & \dot{\mathbf{r}}(\tau) & =v_{\tau} \mathbf{q}_{\tau} \\
\ddot{\mathbf{r}}(0) \cdot \mathbf{T}(0) & =a_{T_{0}}, & \ddot{\mathbf{r}}(\tau) \cdot \mathbf{T}(\tau) & =a_{T_{\tau}} .
\end{array}
$$

Here $\mathbf{q}_{0}=\left(\cos \theta_{0}, \sin \theta_{0}\right)$ and $\mathbf{q}_{\tau}=\left(\cos \theta_{\tau}, \sin \theta_{\tau}\right), v$ is the speed and $a_{T}$ is the tangential acceleration.

The variational optimization problem of Equation 4 is posed in an infinite dimensional space of vector-valued functions $\mathbf{r}(t)$. This problem is numerically solved by choosing a discretization of $\mathbf{r}(t)$ to convert it into a finite-dimensional optimization problem. The discretization should satisfy the boundary conditions.

$\theta$ is not an independent variable but is determined from $x(t)$ and $y(t)$ using Equation 2 when the tangential speed is non-zero. We derive different expressions for $\theta$ at end-points when the tangential speed is zero. For the robot to move in the "forward" direction, the speeds $v_{0}$ and $v_{\tau}$ should be non-negative. Since the optimal trajectory tries to keep the travel time small, it is clear that for the optimal trajectory the tangential speed will never be zero, except perhaps at the end-points if it is given as zero. Thus, $\theta$ will always be welldefined in the interior $(0, \tau)$. The only trouble can arise at the two end-points when the specified tangential speed is zero. The following discussion shows that $\theta$ and its derivatives at end-points can be defined without reparameterizing the curve even when the speed is zero at the end-points.

\section{Different types of boundary conditions}

We want well-defined and computable expressions for $\theta$ and its derivatives in terms of $x(t)$ and $y(t)$ and their derivatives when the tangential speed and acceleration are zero at either of the two end-points. This case is important for specifying tasks that involve starting from rest or coming to rest or both. This gives rise to three different types of boundary conditions which will be discussed shortly.

Assume that third derivatives of $x(t)$ and $y(t)$ exist in a neighborhood of the start and end-points. Let $\left(v_{x}, v_{y}\right)$, $\left(a_{x}, a_{y}\right)$ and $\left(j_{x}, j_{y}\right)$ refer to first, second and third derivatives respectively of $x(t)$ and $y(t)$ with respect to time at $t=0$. Let the subscripts $N$ and $T$ denote the tangential and normal components respectively of a vector. Then,

$$
\begin{aligned}
a_{T} & =a_{x} \cos \theta+a_{y} \sin \theta \\
a_{N} & =-a_{x} \sin \theta+a_{y} \cos \theta \\
j_{T} & =j_{x} \cos \theta+j_{y} \sin \theta \\
j_{N} & =-j_{x} \sin \theta+j_{y} \cos \theta
\end{aligned}
$$

Expand $x(t)$ and $y(t)$ in a Taylor series around $t=0$.

$$
\begin{aligned}
& x(t)=x_{0}+v_{x} t+\frac{1}{2} a_{x} t^{2}+\frac{1}{6} j_{x} t^{3}+\ldots \\
& y(t)=y_{0}+v_{y} t+\frac{1}{2} a_{y} t^{2}+\frac{1}{6} j_{y} t^{3}+\ldots
\end{aligned}
$$

We can expand $\theta$ around $t=0$ in terms of the two Taylor series using Equation 2.

$\theta=\operatorname{atan} 2\left(v_{y}+a_{y} t+\frac{1}{2} j_{y} t^{2}+\ldots, v_{x}+a_{x} t+\frac{1}{2} j_{x} t^{2}+\ldots\right)$

Now, we consider three cases at $t=0$ :

Case 1: Tangential speed $v>0$.

Taking the limit $t=0$ in Equation 8 we have

$$
\theta=\operatorname{atan} 2\left(v_{y}, v_{x}\right) .
$$

Since $v=\sqrt{v_{x}^{2}+v_{y}^{2}}, v>0$ means that at least one of $v_{x}$ and $v_{y}$ is non-zero, and hence $\theta$ is well-defined.

Case 2: Tangential speed $v=0$, tangential acceleration $a_{T} \neq$ 0.

$$
v=0 \Longrightarrow v_{x}=0 \text { and } v_{y}=0 .
$$

Before analyzing this case to get an expression for $\theta$ at end-points, we derive inequality constraints on tangential 
acceleration at end-points when backward motion is not allowed. Consider motion along a straight line. The Taylor expansion of $x(t)$ around $t=0$ when $v=0$ is

$$
x(t)=x_{0}+\frac{1}{2} a_{x} t^{2}+\frac{1}{6} j_{x} t^{3}+\ldots
$$

If $a_{x}<0$, then $x(t)<x_{0}$ for small enough $t$, which is disallowed. Thus $a_{x} \geq 0$ at start-point. Similarly, it can be shown that $a_{x} \leq 0$ at end-point. Generalizing to two dimensions, it is necessary that $a_{T} \geq 0$ at start-point and $a_{T} \leq 0$ at end-point.

We will treat the case where $a_{T}=0$ separately. For now, consider the case where $v=0, a_{T}>0$ if start-point and $a_{T}<0$ if end-point. Substituting $v_{x}=0$ and $v_{y}=0$ in Equation 8, we get

$$
\theta=\operatorname{atan} 2\left(a_{y} t^{2}+\frac{1}{2} j_{y} t^{3}+\ldots, a_{x} t^{2}+\frac{1}{2} j_{x} t^{3}+\ldots\right)
$$

Taking the limit $t=0$ in Equation 11, we have

$$
\theta=\operatorname{atan} 2\left(a_{y}, a_{x}\right) \text {. }
$$

Using Equation 12 in Equation 5 implies that $a_{N}=0$ and $a_{T}=\sqrt{a_{x}^{2}+a_{y}^{2}}>0$. Thus, at least one of $a_{x}$ and $a_{y}$ is non-zero and $\theta$ is well-defined. At the end-point, $a_{T}=-\sqrt{a_{x}^{2}+a_{y}^{2}}<0$ and a similar argument shows that $\theta$ is well-defined.

Case 3 Tangential speed $v=0$ and tangential acceleration $a_{T}=0$.

$$
\begin{gathered}
v=0 \Longrightarrow v_{x}=0 \text { and } v_{y}=0 \text { and } a_{N}=0 . \\
a_{T}=0 \text { and } a_{N}=0 \Longrightarrow a_{x}=0 \text { and } a_{y}=0 .
\end{gathered}
$$

Similar to Case 2, disallowing backward motion leads to constraints on the sign of $j_{T}$ at the end-points. The sign $j_{T}$ can be derived by an argument similar to that for the sign $a_{T}$ in Case 2. At both end-points, $j_{T} \geq 0$. We will treat the case where $j_{T}=0$ separately. For now, consider the case where $v=0, a_{T}=0, j_{T}>0$ at any end-point. Again, following a line of reasoning similar to that for Case 2, we can show that

$$
\theta=\operatorname{atan} 2\left(j_{y}, j_{x}\right)
$$

and that $\theta$ is well-defined. Analysis of further cases, where $j_{T}=0$ is not necessary because we only need to consider input boundary conditions where $v=0$ and $a_{T}=0$.

To summarize, the three types of boundary conditions are:

- Type 1: $v>0$

- Type 2: $v=0, a_{T} \neq 0$. Here $a_{N}=0, a_{T}>0$ if startpoint and $a_{T}<0$ if end-point

- Type 3: $v=0, a_{T}=0$. Here $a_{N}=j_{N}=0$, and $j_{T}>0$ for both end-points.

Obviously, Type 1 is simplest and Equation 2 can be used to express $\theta$. For Type 2, the acceleration vector determines $\theta$. Note that the normal component of acceleration is necessarily zero since speed is zero. For Type 3, the jerk vector determines $\theta$. For this case, the normal component of jerk is zero at the particular end-point. Thus, for $\theta$ to be welldefined, the discretization of $x(t)$ and $y(t)$ should be such that their third-derivatives exist.

\section{Discretization of the problem}

The nonlinear optimization problem Equation 4 is posed on an infinite dimensional space of vector-valued functions $\mathbf{r}(t)$. We minimize $J$ in a finite dimensional subspace by discretizing $x(t)$ and $y(t)$. From Sections II-B and II-C, we see that to completely define the problem we need to specify 4 boundary conditions per end-point per space dimension - position and three derivatives. Hence, we choose heptic interpolating splines as the basis functions. Heptic splines are degree seven piecewise polynomials with continuous derivatives up to order six. As a function, each spline $x(t)$ and $y(t)(M+1$ polynomial pieces) can be uniquely determined from 8 boundary conditions and its value on $M$ interior nodes. In addition to the travel time $\tau$, these nodal function values $\left\{x_{i}, y_{i}\right\}_{i=1}^{M}$ are the parameters that will be found by optimization. The choice of time values $\left\{t_{i}\right\}_{i=1}^{M}$ to which these nodal values correspond will be discussed in Section III.

We need to provide 8 boundary conditions for each spline. In the input specification of Equation 4, only derivatives of up to second order (position, velocity and acceleration) are given. The values of normal acceleration $a_{N}$, tangential jerk $j_{T}$, and normal jerk $j_{N}$ are not provided as input. This is intentional. For example, when speed is non-zero, specifying $a_{N}$ will indirectly specify the curvature, which is difficult to choose. Of course, $a_{N}$ would be zero necessarily if speed is zero. Instead these three quantities $\left(a_{N}, j_{T}\right.$, and $\left.j_{N}\right)$ are left as unknown parameters for the optimization problem. These are determined along with the optimal trajectory. Thus, each end-point has four conditions per space dimension ( $x$ and $y$ ), and if a quantity $\left(a_{N}, j_{T}, j_{N}\right)$ is not specified but can physically be non-zero, then it is left as a parameter to be found by optimization.

Table I lists the three types of boundary conditions, the quantities that can be automatically deduced, and the unknown quantities that must be determined by optimization.

To summarize, if we want to find $M \geq 0$ interior nodes, we will have up to $2 M+1+6$ unknowns to determine. The breakdown is

- $2 M$ for $M$ nodes with 2 unknowns ( $x$ and $y$ components) per node

- 1 unknown $\tau$

- Up to 6 unknowns on boundary. Maximum 3 unknowns $\left(a_{N}, j_{T}, j_{N}\right)$ per end-point.

\section{E. Dimensional analysis and determination of characteristic weights}

The cost functional $J$ is a weighted sum of terms with different physical dimensions. For $J$ to be meaningful as a physical quantity the weights should have appropriate physical dimensions. We will use dimensional analysis to 
TABLE I

THE THREE TYPES OF BOUNDARY CONDITIONS.

\begin{tabular}{|c|c|c|c|c|c|}
\hline Type of BC & Input values & $\theta$ & Deduced values & Unknowns to determine & Remarks \\
\hline \hline Type 1 & $v>0$ & $\operatorname{atan} 2\left(v_{y}, v_{x}\right)$ & - & $a_{N}, j_{N}, j_{T}$ & - \\
\hline Type 2 & $v=0, a_{T} \neq 0$ & $\operatorname{atan} 2\left(a_{y}, a_{x}\right)$ & $a_{N}=0$ & $j_{N}, j_{T}$ & $a_{T}>0$ at start-point and $a_{T}<0$ at end-point \\
\hline Type 3 & $v=0, a_{T}=0$ & $\operatorname{atan} 2\left(j_{y}, j_{x}\right)$ & $a_{N}=0, j_{N}=0$ & $j_{T}$ & $j_{T}>0$ at both end-points \\
\hline
\end{tabular}

determine their form in terms of characteristic physical quantities.

All the physical quantities in the cost functional (time, jerk, acceleration, and angular velocity and acceleration) depend on only two units - length $L$ and time $T$. The input to a motion task specifies the typical length and velocity scales. For example, the characteristic length $L_{*}$ can be taken as the distance between the end-points (or is specified by the user if both the end-points are coincident with different orientations). In addition, the average of starting and stopping speed defines a characteristic speed $V_{*}$. If both are zero, the robot's typical operating speed can be used. The speed $V_{*}$ determines the characteristic time $T_{*}=L_{*} / V_{*}$. We use only the two important scales $\left(L_{*}\right.$ and $\left.V_{*}\right)$. For this analysis, other dimensionless parameters such as ratios of starting and stopping velocities and accelerations will be ignored, since they would make the analysis more complex than necessary.

From Equation 3 we see that $J$ has dimensions $L^{0} T^{1}$ due to the first term $(\tau)$. Thus $w_{T}$ should have dimensions $L^{-2} T^{6}$. Similarly, the dimensions of $w_{N}, w_{\dot{\theta}}$, and $w_{\ddot{\theta}}$ are $L^{-2} T^{6}, L^{0} T^{2}$, and $L^{0} T^{4}$ respectively. Let $\widehat{w}_{T}, \widehat{w}_{N}, \widehat{w}_{\dot{\theta}}, \widehat{w}_{\ddot{\theta}}$ be the characteristic weights. Thus, if the characteristic length of a task is $L_{*}$ and the characteristic time is $T_{*}$, the weight $\widehat{w}_{T}$ should be proportional to $L_{*}^{-2} T_{*}^{6}$. That is, $\widehat{w}_{T} \propto \frac{T_{*}^{6}}{L^{2}}$. Similarly, we can compute the dimensional part of other three weights.

Now we determine the typical values of the characteristic weights by optimizing a few representative motion tasks. Consider a one dimensional motion optimization problem. The robot starts from $x=0$ at $t=0$ and stops at $x=L$ at $t=\tau$. The accelerations are zero at both end-points. Using the minimizing property of quintic splines [11], the quintic polynomial

$$
x(t)=L\left(10\left(\frac{t}{\tau}\right)^{3}-15\left(\frac{t}{\tau}\right)^{4}+6\left(\frac{t}{\tau}\right)^{5}\right)
$$

satisfies these boundary conditions and minimizes $\int_{0}^{\tau} \dddot{x}^{2} d t$ for any fixed $\tau$. For this choice of $x(t)$, it can be computed that

$$
J(\tau)=\tau+720 \frac{\widehat{w}_{T} L^{2}}{\tau^{5}} .
$$

Terms involving other weights are zero because this is a onedimensional motion. Minimizing $J$ as a function of $\tau$ only, we get

$$
1-\frac{1}{3600} \frac{\tau^{6}}{L^{2} \widehat{w}_{T}}=0
$$

Using the characteristic time and length scales, this gives

$$
\widehat{w}_{T}=\frac{1}{3600} \frac{T_{*}^{6}}{L_{*}^{2}} .
$$

$\widehat{w}_{N}$ can then be fixed as a factor times $\widehat{w}_{T}$ where a larger factor implies less tolerance for jerk in the normal direction. Choosing the factor as 1.0, we get

$$
\widehat{w}_{N}=\frac{1}{3600} \frac{T_{*}^{6}}{L_{*}^{2}} .
$$

For computing the remaining weights $\left(\widehat{w}_{\dot{\theta}}\right.$ and $\left.\widehat{w}_{\ddot{\theta}}\right)$, we move on a circle starting from $\theta=0$ and stop at $\theta=2 \pi$. The accelerations at end-points are zero. As before, we choose a quintic polynomial in $\theta$ with $L$ interpreted as $2 \pi$. Minimizing $\tau+\widehat{w}_{\dot{\theta}} \int_{0}^{\tau} \dot{\theta}^{2} d t$ and $\tau+\widehat{w}_{\ddot{\theta}} \int_{0}^{\tau} \ddot{\theta}^{2} d t$ with respect to $\tau$, and using the characteristic length and time scales we get

$$
\begin{aligned}
& \widehat{w}_{\dot{\theta}}=\frac{7}{10(2 \pi)^{2}} T_{*}^{2}, \quad \text { and } \\
& \widehat{w}_{\ddot{\theta}}=\frac{7}{360(2 \pi)^{2}} T_{*}^{4} .
\end{aligned}
$$

Equations 14, 15, and 16 define the characteristic weights as functions of the characteristic length and velocity scales. Each of the four weights is now factored into two parts as

$$
w=f \widehat{w}
$$

where $\widehat{w}$ is the characteristic weight and $f$ is a dimensionless multiplicative factor. The characteristic weight is computed as a function of the length and velocity scales of a given task. The factor $f$ is the part that is varied by a user for customization.

\section{DETAILS OF THE OPTIMIZATION PROCESS}

The discretized function $J$ is a smooth function of the unknowns (Section II-D). The integrals in $J$ are evaluated using eighth order Gaussian quadrature rule on each interval $\left[t_{i}, t_{i+1}\right]$. We use the Newton trust-region method [25] as implemented in TAO/PETSc [26], [27] to minimize $J$. This iterative method requires information about the function value and its first and second derivatives at the chosen iterates. We compute the derivatives using forward mode Automatic Differentiation [28].

\section{A. Choosing an initial guess}

A "reasonable" initial guess for each of the parameters to be optimized is crucial for a good solution of the nonlinear optimization problem. These parameters can be divided into 3 categories - spline nodal values $\left\{x_{i}, y_{i}\right\}_{i=1}^{M}, \tau$, and boundary condition terms $\left(a_{N}, j_{T}, j_{N}\right)$. 
Except for a case mentioned below, the initial guesses of $a_{N}, j_{T}, j_{N}$ are zero. The optimal values would be nonzero in general. Even if we choose them using dimensional analysis, there is no good indicator whether a positive value or a negative value would be good. We make the simplest choice by initializing them to 0 . For Type 3 boundary condition, however, we know the sign of $j_{T}$ at end-points (always positive, Section II-C) and the value is chosen via dimensional analysis. $j_{T}=10 V_{*}^{3} / L_{*}^{2}$. The factor 10 makes the initial travel time small.

Next we guess the initial value of $\tau$. It is chosen using the length and velocity scales. $\tau_{0}=0.75 L_{*} / V_{*}$. The factor 0.75 is chosen to keep the initial guess of $\tau$ small. If the initial travel time is large, the initial trajectory has a tendency to self-intersect instead of forming a non-intersecting path between end-points. This is physically intuitive. What is interesting numerically is that in one of the test cases, this loop did not vanish in the optimization process even when a better non-intersecting trajectory (with smaller $J$ ) existed. See Figure 2(c). We choose a small $\tau$ to avoid this trap of a local minimum.

Finally we guess the nodal values for the two heptic splines. For any fixed $\tau$, consider the two unique heptic polynomials in $t$ that satisfy the 8 boundary conditions on $x(t)$ and $y(t)$ respectively. The values of these polynomials at $\left\{t_{i}\right\}_{i=1}^{M}$ are sampled to obtain the initial guess for $x_{i}$ and $y_{i}$. Of course, we need to choose a good subdivision scheme to fix $t_{i}$ first. Equispaced $t_{i}$ is not a good choice for all boundary conditions. For example, if we have Type 3 boundary conditions (zero velocity and zero acceleration) at both end-points, equispaced $t_{i}$ will lead to nodes that are close together in space. At best, this will make the Hessian ill-conditioned and at worst will make the spline (and thus the trajectory) highly sensitive to changes in nodal values. To avoid this, non-equispaced $t_{i}$ are chosen. They are clustered toward the center $\left(\frac{\tau}{2}\right)$ so that the resulting nodes $x_{i}$ and $y_{i}$ do not cluster towards the start and end points. Numerical results show that this leads to better conditioning and fewer Newton iterations. We skip the details.

\section{RESUlts AND EVAluAtion}

We evaluate our method using a test set consisting of 4 base cases $(A, B, C, D)$ with different boundary conditions as shown in Table II. The optimal paths for all the four cases are shown in Figure 2. For the base cases, the multiplying

TABLE II

BOUNDARY CONDITIONS FOR THE FOUR BASE CASES

\begin{tabular}{|c|c|c|c|c|c|c|}
\hline Case & $(x, y, \theta)_{0}$ & $v_{0}$ & $a_{T_{0}}$ & $(x, y, \theta)_{\tau}$ & $v_{\tau}$ & $a_{T_{\tau}}$ \\
\hline \hline $\mathrm{A}$ & $(0,0,0)$ & 0 & 0 & $(4,2,-\pi / 4)$ & 0 & 0 \\
\hline $\mathrm{B}$ & $(0,0,0)$ & 1 & 0 & $(0,5, \pi / 2)$ & 0.5 & 0 \\
\hline $\mathrm{C}$ & $(0,0,0)$ & 2 & 0 & $(2,0.5, \pi / 4)$ & 1 & -0.5 \\
\hline $\mathrm{D}$ & $(0,0,0)$ & 0 & 0.5 & $(-6,0,-\pi / 2)$ & 0 & -0.5 \\
\hline
\end{tabular}

factors $f$ (Equation 17) corresponding to all the four weights $\left(w_{T}, w_{N}, w_{\dot{\theta}}, w_{\ddot{\theta}}\right)$ are set to 1.0 and the characteristic velocity
$V_{*}$ is chosen to be $0.5 \mathrm{~m} / \mathrm{s}$. The characteristic length $L_{*}$ is the straight line distance between end-points. Each of these cases was run with the number of interior spline nodes taken from the set $\{1,3,7,15\}$ so that the nodes of different refinement levels form a nested sequence. The intent is to see how the optimal value of $J$ decreases as the number of nodes $M$ increases. If the sequence is not nested, optimal $J$ may not reduce even if $M$ is increased. The log-log plot Figure 3 shows that for all four cases, the optimal $J$ reduces as $M$ increases. Ideally the plot should compare $J$ for a finite

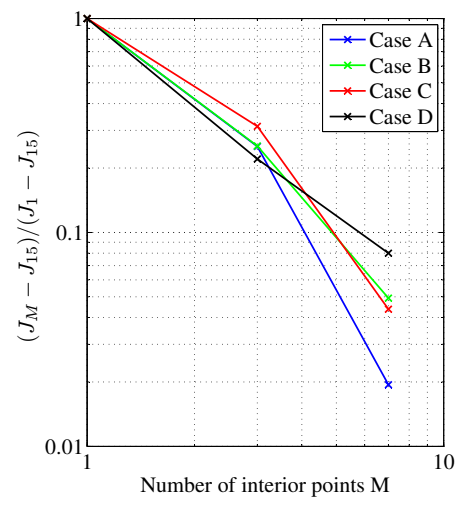

Fig. 3. For each of the cases, the optimal cost decreases as more interior spline nodes are added. Very little change is observed after $M=15$, hence all the other optimal values are compared with $J$ for $M=15$.

$M$ with the exact $J$ when the original continuous problem is solved. Since we do not know the exact minimal value of $J$, the value with largest number of points $M=15$ is taken as the 'exact' value. The optimal $J$ did not change appreciably when $M$ was increased beyond 15 , justifying this approximation.

\section{A. Effect of change of boundary conditions on discomfort}

This section evaluates whether the characteristic weights computed in Section II-E yield similar values of the discomfort measure for varying boundary conditions.

For Case B, the initial and final velocities were simultaneously varied from $0.1 \mathrm{~m} / \mathrm{s}$ to $1 \mathrm{~m} / \mathrm{s}$. For each choice of velocities, the final pose was moved along the $y$ axis such that the straight line distance between the start and end poses varied from $5 \mathrm{~m}$ to $15 \mathrm{~m}$. The characteristic velocity $V_{*}$ was set to $1.0 \mathrm{~m} / \mathrm{s}$.

Average of the discomfort measure is plotted against the boundary conditions in Figure 4. The average discomfort is independent of the length scale of the task. It does have a slight dependence on the starting and stopping velocities. This is because, as discussed in Section II-E, we have not kept all the dimensionless parameters constant while varying the boundary conditions. The slight variation seen in the figure justifies this simplification.

\section{B. Effect of weights on discomfort}

The multiplicative factor $f$ corresponding to each of $w_{T}$, $w_{N}, w_{\dot{\theta}}, w_{\ddot{\theta}}$ was assigned a set of 11 values centered at 1.0 while keeping the other multiplicative factors constant. This 


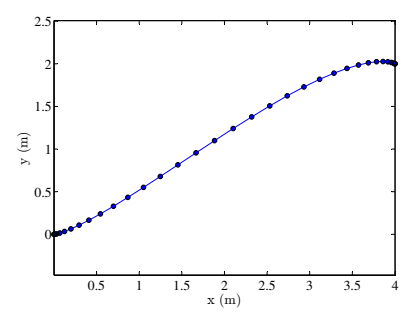

(a) Case A

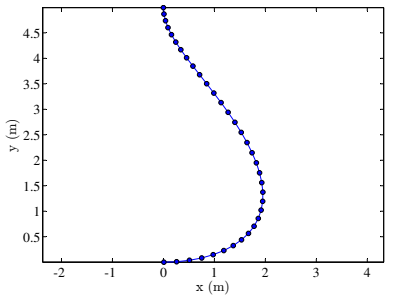

(b) Case B

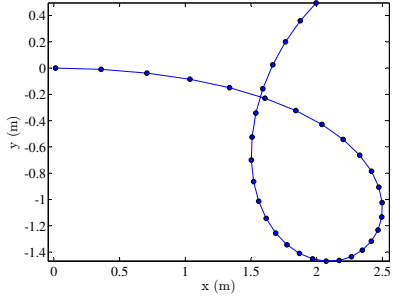

(c) Case $\mathrm{C}$

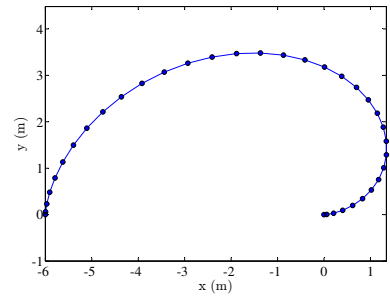

(d) fig:Case D

Fig. 2. Optimal paths for the four base cases shown in Table II. The starting pose for all cases is $(0,0,0)$. The circles are drawn at equal intervals of time. Thus, lesser spacing between circles implies higher speed. Case A has zero speed and acceleration at both ends. As expected, the path is almost a straight line. In Case B, the initial velocity is non-zero and is not directed toward the destination. This results in a more curved path. In case $\mathrm{C}$, we deliberately chose a large initial value of $\tau$. This results in a local optimum with self-intersection. By choosing a smaller initial value, we observed a non-self-intersecting path that had a lower cost (not shown here). In Case D, a non zero starting acceleration directed away from the destination results in a path with relatively high curvature.

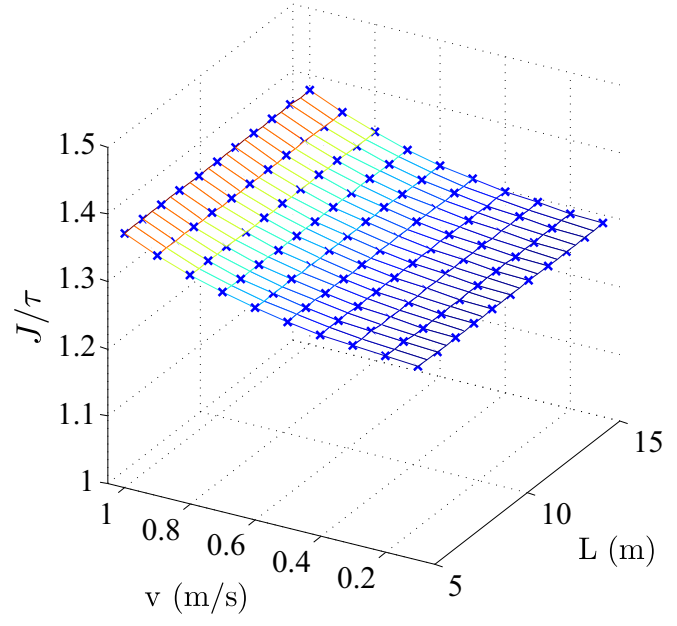

Fig. 4. The average value of the cost functional over the travel duration, plotted against the velocity $v$ at the boundaries and the shortest distance $L$ between the boundary poses.

resulted in 44 cases for each of the 4 base cases of Table II. In total, there are $4 \times 44=176$ test cases. The number of interior nodes $M$ is fixed to 3 . The algorithm converges in all the 176 cases. Except for a few outliers, it takes less than 20 Newton iterations to converge.

As different measures of discomfort, the Root Mean Squared (RMS) values of tangential and normal jerk, tangential and normal acceleration, angular velocity and acceleration, and the total travel time, were analyzed. It was found that all the measures vary uniformly with change in the weights. For ease of discussion, the effect of varying weights on only two measures of discomfort is described, namely the root mean squared tangential jerk, given by

$$
j_{T_{R M S}}=\sqrt{\frac{\int_{0}^{\tau}(\dddot{\mathbf{r}} \cdot \mathbf{T})^{2} d t}{\tau}}
$$

and root mean squared angular acceleration, given by

$$
\ddot{\theta}_{R M S}=\sqrt{\frac{\int_{0}^{\tau} \ddot{\theta}^{2} d t}{\tau} .}
$$

Figure 5(a), shows how $j_{T_{R M S}}, \ddot{\theta}_{R M S}$ and $\tau$ change as

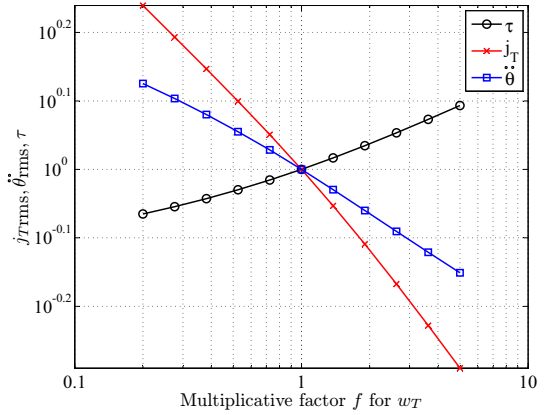

(a) Effect of varying $w_{T}$ for Case $\mathrm{A}$

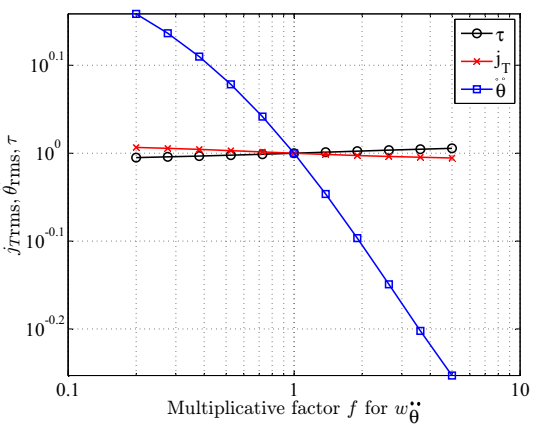

(b) Effect of varying $w_{\ddot{\theta}}$ for Case $\mathrm{D}$

Fig. 5. Effect of varying the weights on measures of discomfort plotted on a log-log scale. The multiplicative factor $f$ for the weights $w_{T}$ and $w_{\ddot{\theta}}$ is varied from 0.2 to 5 in 11 steps in (a) and (b) respectively. This ratio is plotted on the $x$ axis. On the $y$ axis, $\tau$ and the root mean squared (RMS) values of $j_{T}$ and $w_{\ddot{\theta}}$, divided by the corresponding RMS value at the characteristic weight are plotted.

the tangential weight $w_{T}$ varies. From the cost functional (Equation 3), we expect that the largest effect of varying $w_{T}$ should be seen on $j_{T_{R M S}}$, which is borne out by the figure. Thus, qualitatively speaking, motion that has high jerk can be made more comfortable by increasing $w_{T}$. This will result in longer travel time. In Figure 5(b), $w_{\ddot{\theta}}$ is varied. The largest effect of varying $w_{\ddot{\theta}}$ is on $\ddot{\theta}_{R M S}$. Thus, if a user feels that the turns are too uncomfortable, $w_{\ddot{\theta}}$ can be increased for increasing comfort. 
Preliminary analysis of a few cases indicates that the measures of comfort vary with the factor $f$ as a power law. If such an empirical power law can be found, it can form a useful basis for customizing the weights and this analysis will be further pursued in future work. In general, discomfort will be a function of all four weights, and this function could be useful for finer control (if required) of the overall discomfort level.

\section{A note on run-time}

For all the experiments, the algorithm takes 0.5 seconds, on average, on a recent (as of 2009) $1.5 \mathrm{GHz}$ laptop to converge. We expect that optimizing our code will lead to a further reduction in the run-time.

\section{CONCLUSIONS AND FUTURE WORK}

We have formulated a performance measure that quantifies the discomfort of a human user while riding an assistive mobile robot. The performance measure is a weighted sum where the weights can be customized by a user for comfort. We have developed an approach for planning trajectories that satisfy boundary conditions on pose, velocity and acceleration, and minimize the discomfort measure. Results show that our solution method is robust, generalizes to tasks with various boundary conditions, and that solutions can be computed in near real-time. Results also show that weights customized for one task result in similar values of the performance measure in other tasks.

For this paper we have focused solely on planning comfortable trajectories ignored the presence of obstacles. However, obstacle avoidance is an important component in planning trajectories for autonomous robots [29], [30] and this framework will be extended to include obstacles. Further, this framework will be implemented on a wheelchair. Human-subjects evaluation will be performed to determine if the measure of discomfort is appropriate or if further modifications are necessary.

\section{ACKNOWLEDGMENT}

This work has taken place in the Intelligent Robotics Lab at the Artificial Intelligence Laboratory, The University of Texas at Austin. Research of the Intelligent Robotics lab is supported in part by grants from the Texas Advanced Research Program (3658-0170-2007), and from the National Science Foundation (IIS-0413257, IIS-0713150, and IIS0750011).

\section{REFERENCES}

[1] J. Y. Wong, Theory of Ground Vehicles, 4th ed. Wiley-Interscience, 2008.

[2] J. Förstberg, "Ride comfort and motion sickness in tilting trains: Human responses to motion environments in train experiment and simulator experiments," Ph.D. dissertation, KTH Royal Institute of Technology, 2000.

[3] S. Iwnicki, Handbook of Railway Vehicle Dynamics. CRC Press, 2006.

[4] J. Reuter, "Mobile robot trajectories with continuously differentiable curvature: An optimal control approach," in IEEE/RSI Conference on Intelligent Robots and Systems, vol. 1, 1998, pp. 38 - 43.
[5] T. Fraichard and A. Scheuer, "From reeds and shepp's to continuouscurvature paths," IEEE Transactions on Robotics and Automation, vol. 20, no. 6, pp. 1025-1035, 2004.

[6] C. G. L. Bianco and M. Romano, "Smooth motion generation for unicycle mobile robots via dynamic path inversion," IEEE Transactions on Robotics, vol. 20, no. 5, pp. 884 - 891, 2004.

[7] — "Bounded velocity planning for autonomous vehicles," in IEEE/RSJ International Conference on Intelligent Robots and Systems, 2005, pp. 685-690.

[8] H. Seki and S. Tadakuma, "Velocity pattern generation for power assisted wheelchair based on jerk and acceleration limitation," in 31st Annual Conference of IEEE Industrial Electronics Society, 2005.

[9] A. Piazzi, C. G. L. Bianco, and M. Romano, " $\eta^{3}$ splines for the smooth path generation of wheeled mobile robots," IEEE Transactions on Robotics, vol. 23, no. 5, 2007.

[10] S. Gulati and B. Kuipers, "High performance control for graceful motion of an intelligent wheelchair," in IEEE International Conference on Robotics and Automation, 2006, pp. 3932-3938.

[11] T. Flash and N. Hogan, "The co-ordination of arm movements: an experimentally confirmed mathematical model," The Journal of Neuroscience, vol. 5, pp. 1688-1703, 1985.

[12] E. Todorov and M. Jordan, "Smoothness maximization along a predefined path accurately predicts the speed profiles of complex arm movements," Journal of Neurophysiology, vol. 80, no. 2, pp. 696-714, 1998.

[13] M. Žefran and V. Kumar, "Planning of smooth motions on SE(3)," in IEEE International Conference on Robotics and Automation, vol. 1, 1996, pp. 121-126.

[14] H. L. Langhaar, Dimensional Analysis and Theory of Models. Wiley, 1951.

[15] J. L. Troutman, Variational Calculus and Optimal Control: Optimization with Elementary Convexity, 2nd ed. Springer, 1995.

[16] L. E. Dubins, "On curves of minimal length with a constraint on average curvature and with prescribed initial and terminal positions and tangents," American Journal of Mathematics, vol. 79, pp. 497$516,1957$.

[17] J. A. Reeds and L. A. Shepp, "Optimal paths for a car that goes both forward and backward," Pacific Journal of Mathematics, vol. 145, no. 2, pp. 367-393, 1990.

[18] D. J. Balkcom and M. T. Mason, "Time optimal trajectories for differential drive vehicles," International Journal of Robotics Research, vol. 21, no. 3, pp. 199-217, 2002.

[19] C. Fernandes, L. Gurvits, and Z. X. Li, "A variational approach to optimal nonholonomic motion planning," in IEEE International Conference on Robotics and Automation, 1991, pp. 680-685.

[20] J. E. Bobrow, B. Martin, G. Sohl, E. C. Wang, F. C. Park, and J. Kim, "Optimal robot motions for physical criteria," Journal of Robotic Systems, vol. 18, no. 12, pp. 785-795, 2001.

[21] T. M. Howard and A.Kelly, "Optimal rough terrain trajectory generation for wheeled mobile robots," The International Journal of Robotics Research, vol. 26, no. 2, pp. 141-166, 2007.

[22] G. Arechavaleta, J.-P. Laumond, H. Hicheur, and A. Berthoz, "An optimality principle governing human walking," IEEE Transactions on Robotics, vol. 24, pp. 5-14, 2008.

[23] J. T. Betts, Practical Methods for Optimal Control Using Nonlinear Programming. SIAM : Advances in Control and Design Series, 2001.

[24] D. Ferguson, T. M. Howard, and M. Likhachev, "Motion planning in urban environments: Part I," in IEEE/RSJ International Conference on Intelligent Robots and Systems, 2008, pp. 1063-1069.

[25] A. R. Conn, N. I. M. Gould, and P. L. Toint, Trust-Region Methods. Society for Industrial and Applied Mathematics, Philadelphia, PA, 2000.

[26] S. Benson, L. C. McInnes, and J. J. Moré, "TAO users manual," Mathematics and Computer Science Division, Argonne National Laboratory, Tech. Rep. ANL/MCS-TM-242, 2000. [Online]. Available: http://www-unix.mcs.anl.gov/tao/docs/tao_manual.pdf

[27] S. Balay, W. D. Gropp, L. C. McInnes, and B. F. Smith, "PETSc users manual," Mathematics and Computer Science Division, Argonne National Laboratory, Tech. Rep. ANL-95/11 - Revision 2.1.1, 2001. [Online]. Available: http://www.mcs.anl.gov/petsc/petscas/snapshots/petsc-current/docs/manual.pdf

[28] A. Griewank and A. Walther, Evaluating Derivatives: Principles and Techniques of Algorithmic Differentiation, 2nd ed. SIAM, 2008.

[29] J.-C. Latombe, Robot Motion Planning. Kluwer Academic, 1991.

[30] S. LaValle, Planning Algorithms. Cambridge University Press, 2006. 